\title{
DOPAMINE AGONIST IN TREATMENT OF ADHD WITH RESTLESS LEGS SYNDROME AND ODD
}

A 6-year-old male with attention deficit hyperactivity disorder who responded poorly to methylphenidate (MPH) was benefited following treatment with the dopamine agonist ropinirole, in a report from the Hopital Robert Debre, Paris, France. Restless legs syndrome and sleep problems were comorbid disorders that were linked to iron deficiency (serum ferritin $10 \mathrm{mcg} / \mathrm{L}$ ) and partially relieved by a 3-month course of ferrous sulfate. Symptoms of ADHD and oppositional behavior were also improved initially but later relapsed, despite a normal ferritin level $(73 \mathrm{mcg} / \mathrm{L})$ and an increased dose of MPH. Ropinirole $(0.25$ to $0.5 \mathrm{mg}$, 90 min before bedtime) resulted in improvements in sleeping habits, ADHD and ODD, control of restless leg movements, and a reduction in the dose of MPH. No adverse effects were observed. (Konofal E, Arnulf I, Lecendreux M, Mouren M-C. Ropinirole in a child with attention-deficit hyperactivity disorder and restless legs syndrome. Pediatr Neurol May 2005;32:350-351). (Respond: Dr Konofal, Service de Psychopathologie de l'Enfant et de l'Adolescent, Hopital Robert Debre, 48, bd Serrurier, 75020 Paris, France).

COMMENT. Having failed to diagnose restless legs syndrome in my patients with $\AA \mathrm{DHD}$, despite polysomnograms in many, I was surprised to read the authors' reference to an incidence of up to $40 \%$. The potential benefit of a dopamine agonist in the treatment of ADHD unresponsive to MPH is of interest and warrants further study.

\section{COGNITIVE DEFICITS IN CHILDREN WITH MULTIPLE SCLEROSIS}

The association between cognitive functioning and clinical features of 37 children (ages 8-17; mean 14.8 years) with a diagnosis of multiple sclerosis (MS) is reported from the State University of New York at Stony Brook, NY. Thirteen $(35 \%)$ had cognitive impairment, especially in attention, memory and language, and required academic assistance in school. The majority was on disease modifying drug therapy, and $48 \%$ suffered from fatigue. Six of 13 given a structured psychiatric evaluation had an affective disorder. The degree of disability, number of relapses, total disease length, and age at onset were correlated with cognitive dysfunction. At one year follow-up in 8 patients, 5 showed progressive cognitive decline. (MacAllister WS, Belman AL, Milazzo M et al. Cognitive functioning in children and adolescents with multiple sclerosis. Neurology April (2 of 2) 2005;64:14221425). (Reprints: Dr Lauren B Krupp, State University of New York at Stony Brook, HSC T12 020, Department of Neurology, Stony Brook, NY 11794).

COMMENT. Similar to findings in adults, children and adolescents with MS have a high incidence of cognitive impairment and decline, especially affecting attention, memory and language. Cognitive dysfunction involving memory, language, and visual perception is included among initial symptoms of MS in a report of 5 childhood cases (Iannetti P et al, 1996; Progress in Pediatric Neurology III, PNB Publ, 1997;p553). 